\title{
A Robótica Educativa no Ensino de Lógica de Programação: uma revisão sistemática da literatura
}

Francisco Euder dos Santos - PPGIE/UFRGS, IFRO, euder@ifro.edu.br

Dauster Souza Pereira - PPGIE/UFRGS, IFRO, dauster@ifro.edu.br Juliana Martins Godin - CEFET-MG, julianagodin@gmail.com José Valdeni De Lima - PPGIE/UFRGS, valdeni@inf.ufrgs.br

Milton Antônio Zaro - PPGIE/UFRGS, zaro@ufrgs.br

Alberto Bastos do Canto Filho - PPGIE/UFRGS, alberto.canto@ufrgs.br

Resumo: Este artigo tem como objetivo apresentar o resultado de uma Revisão Sistemática da Literatura (RSL) dos trabalhos relacionados à utilização da Robótica Educativa no processo ensino-aprendizagem da disciplina Lógica de Programação no Brasil, publicados em artigos, dissertações e teses, entre 2013 e 2017. Para que a RSL fosse realizada, definiu-se algumas questões norteadoras e, aplicou-se critérios de inclusão e exclusão, com o propósito de selecionar, extrair e apresentar informações dos trabalhos. Os resultados obtidos mostraram como a Robótica Educacional vem sendo conduzida no ensino médio, quais são os softwares e os kits educacionais utilizados na programação dos robôs.

Palavras-chave: Robótica Educativa. Lógica de Programação. Educação.

\section{The Educational Robotics in Programming Logic Teaching: a systematic review of the literature}

Abstract: This paper aims to present the results of a Systematic Review of Literature (RSL) of the works related to the use of Educational Robotics in the teaching-learning process of the discipline Logic of Programming in Brazil, published in papers, dissertations and theses between 2013 and 2017. For RSL to be carried out, some guiding questions were defined and inclusion and exclusion criteria were applied, with the purpose of selecting, extracting and presenting information about the work. The results obtained showed how educational robotics is being conducted in high school, which are the software and educational kits used in robot programming.

Keywords: Educational Robotics. Programming logic. Education.

\section{INTRODUÇÃO}

Atualmente vive-se a era do conhecimento permeado por diversas inovações tecnológicas, proporcionando ao ser humano a possibilidade de apropriar-se e transformálas para atender suas necessidades econômicas, políticas, sociais, dentre outras. Entretanto, mesmo vivendo em uma sociedade em que a informação é difundida e divulgada em tempo real, encontram-se diversos sujeitos à margem das informações construídas ao longo do tempo. Esses sujeitos acabam sendo rotulados pela sociedade como os excluídos tecnológicos. Assim, acredita-se que o papel da escola é permitir o acesso de uma maneira significativa à informação, buscando combater essa exclusão ao mesmo tempo que prepara o sujeito para interagir com os diversos recursos existentes.

Uma dessas inovações tecnológicas que vem crescendo de maneira rápida é a robótica (BARBOSA, 2016). A robótica está inserida em vários ramos de atividade, como 
indústria automobilística, têxtil, agronegócio, alimentícia, entretenimento, dentre outras. No meio acadêmico ela é conhecida como Robótica Educacional (RE) ou Robótica Educativa. A Robótica Educacional tem como objetivo proporcionar aos alunos a compreensão de conceitos trabalhados em sala de aula, na forma prática (CABRAL, 2010). Para Menezes e Santos (2015), a RE pode ser caracterizada como um ambiente de aprendizagem. Segundo o DCNEM (2012), um ambiente de aprendizagem deve permitir a construção de novos saberes, buscando promover a capacidade de aprendizagem permanente do aluno, ao mesmo tempo que sejam desenvolvidas atividades sociais privilegiando o convívio humano.

A proposta do termo RE vem sendo utilizado recentemente na literatura. No Brasil, esse paradigma começou a aparecer em alguns trabalhos em 2007. Porém, compreende-se que a aplicabilidade de protótipo para resolver exercícios de programação tenha surgido com a proposta de Seymour Papert ${ }^{1}$ na década de 1980 nos Estados Unidos.

Na área da Educação, a RE é direcionada para se tornar um novo mediador no processo de ensino-aprendizagem e não na produção de robôs como foco principal (D'ABREU, 1999). Segundo Menezes e Santos (2015), a sua aplicabilidade na educação permite a interdisciplinaridade entre diversas áreas do conhecimento.

A Robótica Educacional é aplicada em várias disciplinas dos cursos técnicos de ensino médio voltados para a área de informática. Essas disciplinas utilizam a RE para desenvolver seus conteúdos programáticos, aliando seus conceitos teóricos com a prática, proporcionado ao aluno um ambiente mais dinâmico durante o processo de ensinoaprendizagem. Uma dessas disciplinas é a Lógica de Programação, que em geral é ministrada no primeiro ano dos Cursos Técnicos em Informática. Essa disciplina é considerada de grande importância na grade curricular, pois é responsável em apresentar o mundo da programação de computadores para os alunos. Entretanto, ela se tornou um objeto de estudo por configurar como umas das disciplinas que tem um grande índice de evasão e reprovação (FARIA, 2013).

Assim, acredita-se que os estudos para acompanhar a constante evolução da informática na Educação é essencial para que os pesquisadores possam conhecer, compreender e aplicar essas inovações tecnológicas em sala de aula. Desta forma, buscando minimizar cada vez mais as lacunas entre aprendizagem e conhecimento, permitindo ao mesmo tempo, que os alunos possam desenvolver a sua criatividade e a capacidade de gerenciar os problemas.

Com base nas considerações feitas anteriormente, decidiu-se realizar uma Revisão Sistemática da Literatura, tendo como motivação a utilização da Robótica Educacional na disciplina Lógica de Programação, buscando compreender quais são os softwares utilizados, kits robóticos e as suas aplicabilidades na condução da disciplina. A pesquisa foi realizada com base em publicações de artigos nacionais, dissertações e teses, datados entre 2013 e 2017.

Este artigo organiza-se em quatro seções, compreendendo a introdução, como a primeira, apresentando a contextualização sobre o tema da pesquisa; na segunda, será apresentado a metodologia utilizada na realização da pesquisa, identificando as questões de pesquisa e as fontes utilizadas; na terceira, apresentam-se os resultados encontrados com base nas pesquisas analisadas; e quarta, apresentam-se as conclusões e as considerações finais.

\footnotetext{
1 Seymour Papert (1928, 2016) foi um matemático e educador estadunidense que lecionava no Massachusetts Institute of Technology (MIT). Ele propôs o termo construcionismo como sendo a abordagem do construtivismo que permite ao educando construir o seu próprio conhecimento por intermédio de alguma ferramenta, como o computador, por exemplo.
}

V. $16 \mathrm{~N}^{\mathrm{o}} 1$, julho, 2018 


\section{METODOLOGIA}

Nesta seção apresenta-se a metodologia utilizada, as questões da pesquisa, estratégia de busca e as aplicações dos critérios de inclusão e exclusão.

\subsection{Procedimentos Metodológicos}

De acordo com Kitchenham (2007), uma revisão sistemática da literatura permite identificar, avaliar e interpretar uma pesquisa buscando explorar determinada questão dentro da pesquisa, área temática ou fenômeno de interesse. Segundo a autora, especificar as questões norteadoras da pesquisa é parte fundamental para qualquer revisão sistemática. Para Prodanov (2013), com a revisão de literatura, o pesquisador mantém contato direto com o material escrito sobre o assunto da pesquisa, desta forma, é possível reportar e avaliar o conhecimento produzido.

Compreende-se que, a partir de questões norteadores é possível determinar os critérios de consulta bibliográfica e, em seguida, realizar o levantamento com a finalidade de selecionar os trabalhos que serão utilizados como parte da análise. Kitchenham (2007) acrescenta que, para realizar uma revisão sistemática é necessário estabelecer previamente critérios de inclusão e exclusão, com o foco na questão da pesquisa. Desta forma, foi aplicado um protocolo norteador para a coleta de dados, como será visto a seguir.

\subsection{Questões da Pesquisa}

A definição das questões da pesquisa é apontada como uma das etapas essenciais para a Revisão Sistemática de Literatura, pois elas permitem que o pesquisador possa conduzir a busca de trabalhos relevantes e necessários (KITCHENHAM, 2007; PRODANOV, 2013). Dessa forma, o objetivo desta pesquisa é responder a seguinte questão central: Como a Robótica Educativa pode contribuir para o ensinoaprendizagem dos conteúdos da disciplina de Lógica de Programação?

Para que fosse possível responder à questão central, adotou-se as seguintes questões da pesquisa:

Q1: Quais são os softwares utilizados na Robótica Educativa?

Q2: Quais são os kits de robótica utilizados na Robótica Educativa?

Q3: Quais são as estratégias didáticas utilizadas na condução da disciplina?

\subsection{Estratégia de Busca}

A consulta inicial foi realizada buscando os trabalhos publicados nos anos de 2013 até 2017 em bibliotecas digitais (Periódicos da Capes, Google Acadêmico, Lume repositório digital da UFRGS, Anais do Workshop de Informática na Escola - WIE, e Simpósio Brasileiro de Informática na Educação - SBIE), em que os títulos, resumos, ou parte do trabalho apresentasse os seguintes critérios: "Robótica Educativa", "Robótica Educacional", "Lógica de Programação", "Linguagem de Programação", "Ensino Médio".

Os critérios adotados foram estruturados na seguinte string de busca: ((“Robótica Educativa" OR "Robótica Educacional") AND ("Lógica de Programação" OR “Linguagem de Programação” OR “Algoritmo”) AND (“Ensino Médio”)).

V. $16 \mathrm{~N}^{\mathrm{o}} 1$, julho, 2018 
Percebeu-se que durante as pesquisas preliminares, os termos "robótica educativa" e "robótica educacional" eram utilizados para representar a mesma ideia; e "Linguagem de Programação" e "Lógica de Programação" em algumas instituições de ensino eram utilizadas para atender ao mesmo objetivo, o ensino dos conteúdos de lógica de programação.

Utilizou-se uma string de busca adaptada para cada biblioteca digital de maneira que os critérios determinados anteriormente fossem atendidos.

\subsection{Aplicações dos Critérios de Inclusão/Exclusão}

Aplicar os critérios de Inclusão e Exclusão permite que o pesquisador possa ter trabalhos que responderão aos objetivos da pesquisa (KITCHENHAM, 2007).

Para o processo de inclusão dos trabalhos selecionados, aplicou-se o seguinte critério: o trabalho deveria responder pelo menos uma das questões da pesquisa.

Para o processo de exclusão as seguintes diretivas foram aplicadas:

- Trabalhos que não atendam aos critérios aplicados de inclusão;

- Trabalhos que não foram aplicados na disciplina Lógica de Programação ou disciplinas afins no Ensino Médio;

- Trabalhos em que aplicabilidade não foi na área da Educação;

- Trabalhos em que a aplicabilidade ocorreu fora do Brasil;

- Trabalhos duplicados em diferentes fontes;

- Trabalhos que não são pesquisas acadêmicas completas (slides, resumos, dentre outros);

- Trabalhos que apresentem somente recomendações ou orientações para a utilização da robótica na educação.

Na seção seguinte serão apresentados os resultados obtidos a partir das consultas realizadas nas bases de dados levando em considerações os critérios de inclusão e exclusão previamente definidos.

\section{RESULTADOS}

Nesta seção são apresentados os resultados encontrados na Revisão Sistemática da Literatura a partir da análise dos trabalhos pesquisados.

\subsection{Extração das Informações}

Durante a busca dos trabalhos para o levantamento das produções cientificas desta RSL, detectou-se algumas revisões realizadas nos últimos anos, Tabela 1. Assim, decidiuse, aplicando as questões desta pesquisa e os critérios de inclusão e exclusão, primeiramente, compreender como estava sendo conduzido o processo de ensinoaprendizagem até aquele momento.

Tabela 1: Revisões sistemáticas da literatura

\begin{tabular}{|l|l|c|c|}
\hline Id & \multicolumn{1}{|c|}{ Título } & \multicolumn{1}{|c|}{ Autores } & Ano \\
\hline 1 & $\begin{array}{l}\text { Robótica na Educação: } \\
\text { Uma Revisão Sistemática dos Últimos } \\
\text { 10 Anos }\end{array}$ & Ranulfo Plutarco Bezerra et al. & 2015 \\
\hline
\end{tabular}




\begin{tabular}{|l|l|l|c|}
\hline 2 & $\begin{array}{l}\text { Robótica Pedagógica Aplicada ao } \\
\text { Ensino de Programação: Uma Revisão } \\
\text { Sistemática da Literatura }\end{array}$ & $\begin{array}{l}\text { Thais Oliveira Almeida, José } \\
\text { Francisco de Magalhães Netto }\end{array}$ & \\
\hline 3 & $\begin{array}{l}\text { O Avanço das Publicações sobre a } \\
\text { Robótica Educacional como possível } \\
\text { Potencializadora no Processo de } \\
\text { Ensino-Aprendizagem: uma Revisão } \\
\text { Sistemática da Literatura }\end{array}$ & $\begin{array}{l}\text { Edjane Mikaelly Silva de } \\
\text { Azevêdo, Deise Juliana }\end{array}$ & Francisco, \\
Albino Oliveira Nunes & \\
\hline 4 & $\begin{array}{l}\text { Revisão Sistemática de Literatura sobre } \\
\text { o Pensamento Computacional por Meio } \\
\text { de Objetos de Aprendizagem }\end{array}$ & $\begin{array}{l}\text { Joethe Moraes de Carvalho et } \\
\text { al. }\end{array}$ & 2017 \\
\hline
\end{tabular}

O Gráfico 1 apresentado a seguir demonstra o resultado da análise das 4 revisões sistemáticas citadas anteriormente destacando o quantitativo de documentos encontrados e selecionadas de cada uma delas.

Gráfico 2: Análise das revisões sistemáticas encontradas

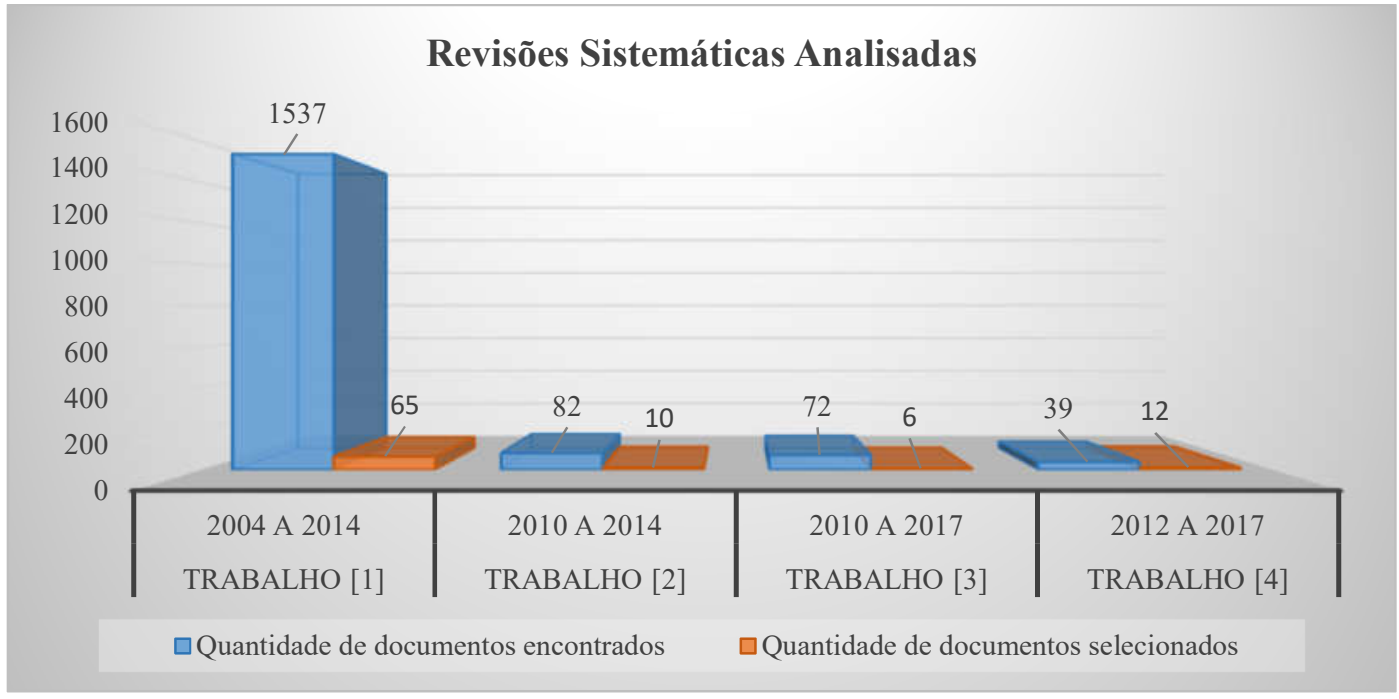

No trabalho [1], através dos critérios estabelecidos anteriormente, identificou-se que apenas 4 documentos eram voltados para a Lógica - enquanto Área de Conhecimento e; $15 \%$ dos robôs eram construídos utilizando a plataforma Arduino e $30 \%$ a plataforma Lego MindStorms. Infelizmente este trabalho não relacionou os documentos pesquisados.

Nos trabalhos [2], [3] e [4], aplicando as questões desta pesquisa e os critérios de inclusão e exclusão, nenhum documento atendeu os requisitos da RSL estabelecida.

Desta forma, prosseguiu-se com RSL proposta. Após inserir a string e o intervalo de publicação nas bibliotecas digitais para a seleção dos trabalhos, obteve-se um rol de 288 trabalhos. Após a leitura dos títulos e resumos dos trabalhos, aplicando as diretivas dos critérios de inclusão e exclusão, obteve-se um total de 40 trabalhos a serem analisados. Esses trabalhos foram classificados em artigos, dissertações e teses, conforme pode ser visualizado na Tabela 2.

Tabela 3: Extração de dados

\begin{tabular}{|l|r|}
\hline Tipo de Trabalho & Quantidade \\
\hline Artigos & 24 \\
\hline
\end{tabular}




\begin{tabular}{|l|r|}
\hline Dissertações & 12 \\
\hline Teses & 04 \\
\hline Total & $\mathbf{4 0}$ \\
\hline
\end{tabular}

Os 40 trabalhos selecionados tiveram seus textos examinados na íntegra e, aplicando novamente os critérios de inclusão e exclusão, teve como resultado 07 estudos selecionados (01 publicado em 2014, 03 em 2015, 02 em 2016 e 01 em 2017) para serem analisados. Esses trabalhos foram reclassificados, conforme pode ser visualizado na Tabela 3.

Tabela 4: Extração de dados após a reclassificação

\begin{tabular}{|l|r|}
\hline Tipo de Trabalho & Quantidade \\
\hline Artigos & 05 \\
\hline Dissertações & 02 \\
\hline Total & $\mathbf{0 7}$ \\
\hline
\end{tabular}

Nos trabalhos que foram excluídos desta RSL, detectou-se, que a RE era aplicado na Educação Infantil, Ensino Fundamental, Graduação em Informática, Matemática e Física; e alguns trabalhos, mesmo apresentando conteúdos de lógica de programação, se tratavam de oficinas em que o público alvo não eram alunos de ensino médio.

Os trabalhos excluídos utilizam a RE com os seguintes software e plataformas: apenas o Scratch 2 , S4A 3 com o Arduino, Arduino IDE $^{4}$ com Arduino e EV3 Software ${ }^{5}$ com Lego.

A seguir, serão apresentadas as respostas das questões de pesquisa.

\subsection{Discussão das Questões de Pesquisa}

A extração de dados permite selecionar os trabalhos que juntos poderão fornecer respostas possíveis para cada questão da pesquisa. Após a extração de dados os trabalhos foram classificados em duas categorias: Robótica Educativa com Arduino, Robótica Educativa com LEGO. A seguir, as análises e contribuições dos trabalhos no processo de ensino-aprendizagem da disciplina Lógica de Programação.

A Tabela 4 apresenta os trabalhos selecionados com suas respectivas identificações para facilitar a sua referência no decorrer das discussões mostradas a seguir.

Tabela 5: Relação dos trabalhos analisados

\begin{tabular}{|l|l|l|c|c|}
\hline ID & \multicolumn{1}{|c|}{ Título } & Tipo & \multicolumn{1}{c|}{ Autores } & Ano \\
\hline 1 & $\begin{array}{l}\text { RecArd: Robô baseado na plataforma } \\
\text { Arduino como facilitador no processo } \\
\text { de ensino-aprendizagem } \\
\text { multidisciplinar }\end{array}$ & Artigo & João Lucas de Souza Silva et al. & 2014 \\
\hline
\end{tabular}

${ }^{2}$ O Scratch é uma linguagem de programação visual criada no Media Lab do Instituto de Tecnologia de Massachusetts. A programação é realizada com blocos de comandos.

${ }^{3}$ O S4A é uma modificação do Scratch, que proporciona ao programador uma forma mais interativa de programar para o Arduino

${ }^{4} \mathrm{O}$ Arduino IDE é um Software padrão para desenvolvimento com a plataforma Arduino.

${ }^{5}$ IDE utilizada para programar os robôs Lego.

V. $16 \mathrm{~N}^{\circ} 1$, julho, 2018 


\begin{tabular}{|c|c|c|c|c|}
\hline 2 & $\begin{array}{l}\text { Robótica Educativa na aprendizagem } \\
\text { de Lógica de Programação: Aplicação } \\
\text { e análise. }\end{array}$ & Artigo & $\begin{array}{l}\text { Eduardo Cambruzzi, Rosemberg } \\
\text { Mendes de Souza }\end{array}$ & 2015 \\
\hline 3 & $\begin{array}{l}\text { Prática de ensino de Programação de } \\
\text { Computadores com Robótica } \\
\text { Pedagógica e aplicação de Pensamento } \\
\text { Computacional }\end{array}$ & Artigo & $\begin{array}{l}\text { Humberto Augusto Piovesana } \\
\text { Zanetti, Cláudio Luís Vieira } \\
\text { Oliveira }\end{array}$ & 2015 \\
\hline 4 & $\begin{array}{l}\text { Estudo Exploratório sobre o uso da } \\
\text { Robótica Educacional no ensino de } \\
\text { Programação Introdutória }\end{array}$ & Dissertação & André Rachman Dargains & 2015 \\
\hline 5 & $\begin{array}{l}\text { Uma Experiência do Uso do Hardware } \\
\text { Livre Arduino no } \\
\text { Ensino de Programação de } \\
\text { Computadores }\end{array}$ & Artigo & $\begin{array}{l}\text { David Albuquerque, } \text { Vitor } \\
\text { Bremgartner, Hillermann Lima, } \\
\text { Nilmara Salgado }\end{array}$ & 2016 \\
\hline 6 & $\begin{array}{l}\text { Utilização da Plataforma de } \\
\text { Prototipação de Hardware Arduino } \\
\text { como Apoio à Aprendizagem de } \\
\text { Conceitos do Componente Curricular } \\
\text { de Programação }\end{array}$ & Dissertação & Renira Carla Soares & 2016 \\
\hline 7 & $\begin{array}{l}\text { BEER QUIZ: O desenvolvimento de } \\
\text { games para o ensino de } \\
\text { Lógica de Programação através do } \\
\text { Scratch }\end{array}$ & Artigo & Alan Paulo et al. & 2017 \\
\hline
\end{tabular}

\subsubsection{Q1: Quais são os softwares utilizados na Robótica Educativa?}

Essa questão permitiu identificar quais foram os softwares e/ou ambientes de programação utilizados para desenvolver os conteúdos de lógica de programação na aplicação da Robótica Educativa.

Os trabalhos selecionados [3] e [7] utilizaram a linguagem de programação visual Scratch, os trabalhos [1], [5] e [6] utilizaram o Arduino IDE, o trabalho [2] utilizava EV3 Software e o trabalho [4] utilizava o DuinoBlocks ${ }^{6}$.

\subsubsection{Q2: Quais são os kits de robótica utilizados na Robótica Educativa?}

Através desta questão foi possível compreender quais são os kits utilizados nas aulas da disciplina Lógica de Programação.

Os trabalhos selecionados [1], [3], [4], [5], [6] e [7] utilizaram o kit de robótica Arduino e o trabalho [2] utilizou o kit de robótica Lego.

O kit Arduino ${ }^{7}$ aparece como sendo o kit mais utilizado nos trabalhos selecionados. O baixo custo dos componentes do kit Arduino pode ter contribuído para a sua escolha na maioria dos trabalhos analisados em detrimento ao kit Lego ${ }^{\circledR}$ MindStorms ${ }^{8}{ }^{\circledR}$ ou similares.

\footnotetext{
${ }^{6}$ DuinoBlocks é uma Linguagem visual baseada em blocos para programação de placas Arduino.

${ }^{7}$ Arduino é uma plataforma de prototipagem eletrônica de hardware livre utilizadas em várias áreas para o desenvolvimento de experimentos tecnológicos (MCROBERTS, 2015).

${ }^{8}$ LEGO Mindstorms é uma linha do brinquedo LEGO, lançada comercialmente em 1998, voltada para a Educação Tecnológica. Fonte: Lego Home. Disponível em: <http://mindstorms.lego.com>. Acesso em: 02 set. 2017.
} 


\subsubsection{Q3: Quais são as estratégias didáticas utilizados na condução da disciplina?}

Nesta questão o objetivo é identificar como que a RE vem sendo utilizada na reconstrução de conhecimentos junto ao aluno.

No trabalho [1], o robô já estava montado e foi utilizado um smartphone para controlar um robô para desviar de obstáculos. Assim, o papel do aluno era desenvolver o programa para que o robô não batesse no obstáculo posicionado em um local previamente definido. Como resultado, a pesquisa apontou que os alunos conseguiram compreender a teoria proposta quando conhecem a aplicação na prática.

Já no trabalho [2], os alunos foram divididos em grupos e utilizaram um robô padrão para aplicar os conteúdos de lógica de programação. $\mathrm{O}$ resultado da pesquisa mostrou que a utilização de uma metodologia lúdica e interativa, como a robótica, colabora na aprendizagem dos alunos.

A análise do trabalho [3] demonstrou que os conteúdos foram trabalhados utilizando a ferramenta Scratch e um robô já montado. Assim o aluno deveria programar na ferramenta e executar no robô. Os resultados obtidos demonstraram de maneira positiva que é possível utilizar um meio inovador do que o modelo tradicional de ensino.

A estratégia utilizada no trabalho [4] foi aplicado por meio de uma oficina para vários alunos do ensino médio com os conteúdos de computação introdutória. Os alunos participaram ativamente para montar diversos circuitos pré-definidos para resolver as questões propostas. A ferramenta utilizada para programar foi o DuinoBlocks. Os resultados apontaram que a pesquisa colaborou de forma significativa no entendimento de conceitos sobre lógica de programação, construção de algoritmos, estruturas de programação e montagem de circuitos eletrônicos.

A condução da disciplina no trabalho [5] foi feita de modo que os alunos desenvolvessem uma programação utilizando a estrutura condicional se-senão em um cenário montado para essa finalidade. A interação do aluno com o Arduino foi montar um circuito previamente definido pelo professor e alterar valores e observar resultados. Os resultados apontaram que a robótica permite estimular o aprendizado em linguagem de programação.

Já no trabalho [6], os conteúdos de lógica de programação - estrutura de condição e estrutura de repetição - foram aplicados utilizando diversos componentes eletrônicos com a plataforma Arduino. A interação do aluno com o Arduino foi observar um circuito previamente montado pelo pesquisador e descrevê-los em forma textual e pseudocódigo. Os resultados demostraram que a utilização de circuitos simples é mais intuitiva para o aluno descrever a sua solução, tornando assim, um estimulador de aprendizagem.

Por fim, no trabalho [7] foi proposto para os alunos desenvolverem jogos utilizando a ferramenta Scratch como forma de colaborar para o ensino de programação. Os resultados demonstraram que os jogos computadorizados, quando utilizados na educação, proporcionam motivação, desenvolvem hábitos de persistência diante de desafios e melhoram a flexibilidade cognitiva dos alunos.

Na seção 4 serão apresentadas as conclusões da RSL.

\section{CONCLUSÕES}

Este artigo teve como objetivo realizar uma Revisão Sistemática da Literatura (RSL) referente à aplicação da Robótica Educacional (RE) no ensino médio, na disciplina Lógica de Programação, entre os anos de 2013 até 2017. De um total de 288 trabalhos pré-selecionados, 40 foram avaliados e, após a aplicação de questão norteadora e critérios de inclusão e exclusão, 07 trabalhos foram discutidos. 
Através desta RSL, identificou que a ferramenta Arduino é utilizado na maioria dos trabalhos pesquisados e que existe um avanço considerável na utilização da ferramenta Scratch (trabalhos discutidos e os excluídos) no cenário de programação em blocos no ensino-aprendizagem de programação.

Percebeu-se que a RE é desenvolvida em vários ambientes distintos, focada no aluno. Buscando como objetivo principal, minimizar a lacuna entre a aprendizagem e conhecimento, ao mesmo tempo que utiliza estratégias abordando um ensino mais lúdico.

Entretanto, ao analisar a metodologia de Robótica Educativa aplicada no ensino de lógica de programação, constata-se que na maioria, com exceção de um trabalho, restringem-se a modelos prontos, em que o aluno acaba utilizando uma ferramenta construída para uma determinada finalidade, muitas vezes sem compreender como aquela ferramenta foi desenvolvida. Percebe-se que as montagens dos kits de robótica são definidas pelo professor e levadas para sala de aula, não permitindo que o aluno aplique seus conhecimentos prévios.

Como trabalhos futuros, acredita-se, que pesquisas devam ser realizadas, buscando estabelecer estratégias intervencionais, em que aluno possa construir/montar seus robôs em uma interação idealizada para atender as suas necessidades educacionais, dentro de um contexto de uma aprendizagem significativa, voltada para a realidade do aluno.

\section{AGRADECIMENTOS}

Ao Instituto Federal de Educação, Ciência e Tecnologia de Rondônia (IFRO), Campus Porto Velho Zona Norte e Campus Cacoal pelo incentivo à produção intelectual.

\section{REFERÊNCIAS BIBLIOGRÁFICAS}

BARBOSA, Fernando da Costa. Rede de Aprendizagem em Robótica: uma perspectiva educativa de trabalho com jovens. 2016. 366 f. Tese (Doutorado em Educação) Universidade Federal de Uberlândia, Uberlândia, 2016. Disponível em: < https://repositorio.ufu.br/handle/123456789/17564 >. Acesso: 03 set. 2017.

BRASIL. Conselho Nacional de Educação (CNE). Diretrizes Curriculares Nacionais para a Educação Profissional Técnica de Nível Médio. Brasília: Ministério da Educação, 2012. Disponível em:< http://portal.mec.gov.br/index.php?option $=$ com_docman\&view $=$ download \&alias $=1166$ 3-rceb006-12-pdf\&category_slug=setembro-2012-pdf\&Itemid=30192>. Acesso em: 03 set. 2017.

CABRAL, Cristiane Pelisolli. Robótica Educacional e Resolução de Problemas: uma abordagem microgenética da construção do conhecimento. Dissertação (Mestrado em Educação) - Programa de Pós-Graduação em Educação, UFRGS, Porto Alegre, 2010. Disponível em: <http:/www.lume.ufrgs.br/handle/10183/29314>. Acesso em: 01 set. 2017.

D'ABREU, João Vilhete Viega. Desenvolvimento de ambientes de aprendizagem baseados no uso de dispositivos robóticos. Anais do X Simpósio Brasileiro de Informática na Educação. Curitiba-PR, 1999. Disponível em:< http://www.conhecer.org.br/download/cp/NOVAS\%20TECNOLOGIAS/M1/leitura\%20 anexa\%204.pdf>. Acesso em: 01 set. 2017. 
FARIA, Eliézer Marques. A Contribuição da Teoria Histórico-Cultural de Vygotsky para o Ensino e Aprendizagem de Algoritmo. Tese (Doutorado em Educação) Pontifícia Universidade Católica de Goiás. Goiânia - GO, 2013. Disponível em: < http://tede2.pucgoias.edu.br:8080/handle/tede/698>. Acesso em: 20 set. 2017.

MALIUK, Karina Disconsi. Robótica Educacional como Cenário Investigativo nas Aulas de Matemática. Dissertação. (Mestrado em Ensino de Matemática) - Instituto de Matemática, Programa de Pós-Graduação em Ensino de Matemática, UFRGS, Porto Alegre, 2009. Disponível em: < http://www.lume.ufrgs.br/handle/10183/17426>. Acesso em: 01 set. 2017.

MCROBERTS, Michael. Arduino Básico. São Paulo: Novatec, 2015.

MENEZES, Ebenezer Takuno de; SANTOS, Thais Helena dos. Verbete robótica educacional. Dicionário Interativo da Educação Brasileira - Educabrasil. São Paulo: Midiamix, 2015. Disponível em: <http://www.educabrasil.com.br/roboticaeducacional/>. Acesso em: 03 set. 2017.

PAPERT, Seymour. A Máquina das Crianças: repensando a escola na era da informática. Porto Alegre. Artes Médicas, 1994.

PRODANOV, Cleber Cristiano; Freitas, Ernani Cesar. Metodologia do Trabalho Científico: Métodos e Técnicas da Pesquisa e do Trabalho. 2. ed. Novo Hamburgo: Feevale, 2013.

SILVEIRA JÚNIOR, C. R.; COELHO, J. D.; SANTOS, L. S. Robótica nas aulas de matemática do ensino médio: uma proposta educacional e de baixo custo. Experiências em Ensino de Ciências (UFRGS) v. 12, p. 82-104, 2017.

KITCHENHAM, Barbara. Guidelines for Performing Systematic Literature Reviews in Software Engineering. EBSE Technical Report. 2007. Disponível em: < https://www.elsevier.com/_data/promis_misc/525444systematicreviewsguide.pdf $>$.

Acesso em: 11 abr. 2018. 